\title{
SOS Response and Staphylococcus aureus: Implications for Drug Development
}

\author{
Luís Cláudio Nascimento da Silva, \\ Roseane Costa Diniz, \\ Isana Maria de Souza Feitosa Lima, \\ Camilla Itapary dos Santos, Matheus Silva Alves, \\ Larissa Isabela Oliveira de Souza and \\ Andrea de Souza Monteiro
}

Additional information is available at the end of the chapter

http://dx.doi.org/10.5772/65960

\begin{abstract}
Damage in genetic material is induced through the action of several drugs (directly or indirectly). Specially, antimicrobials from quinolone class (such as ciprofloxacin) induce DNA damage that promotes the formation of the RecA filament leading to auto-cleavage of LexA and allows the expression of SOS genes, including the error-prone polymerase (like $u m u \mathrm{C}$ ). The SOS pathway plays a critical role in the acquisition of mutations that lead to the emergence of antibiotic-resistant bacteria and the spread of virulence factors. This chapter provides a comprehensive review about the SOS response of Staphylococcus aureus and the modulatory effects of new compounds (natural or synthetics) on this pathway. The effects of some SOS inhibitors are highlighted such as baicalein and aminocoumarins. Compounds able to prevent SOS response are extremely important to develop new combinatory approaches to inhibit $S$. aureus mutagenesis. The study of new SOS inductors could reveal new insights into the pathways used by $S$. aureus to acquire drug resistance; examples of these compounds are the lysine-peptoid hybrid LP5, cyclic peptide inhibitors, etc. These studies can impact the development of new drugs. In conclusion, we hope to provide essential information about the effects of compounds on SOS response from S. aureus.
\end{abstract}

Keywords: DNA damage, mutagenesis, virulence factors, small colony variants 


\section{Introduction}

Drug resistance is a well-known problem involved in the treatment of bacterial infections and its incidence is escalating in an alarming rate [1-3]. As a result, microbial diseases are still among the most serious problems to public health system, especially in developing countries where infectious diseases still represent a major cause of human mortality. Especially alarming is the rapid global spread of multiresistant bacteria that cause common infections and that resist treatment with existing antimicrobial medicines $[4,5]$. The classical paradigm suggests that antibiotic resistance emerges by selection of preexisting mutants in the bacterial population exposed to antibiotics [1]. In contrast, recent data suggested that mutations evolve after cells encounter antibiotic therapy. This kind of mutation is known as adaptive mutation, which is activated by the SOS DNA repair and mutagenesis pathways [6, 7].

The SOS system is the bacterial DNA damage response that is activated by DNA damage or stalled DNA replication caused by the exposure of bacteria to stressful conditions [8] such as antibiotic treatment [9], starvation [10], and oxidative stress [11]. Repair of damaged DNA is critical for bacterial survival, and during this process, some mutations may be introduced into the genome, which may result in bacterial drug resistance $[12,13]$. Accumulation of singlestranded DNA (ss-DNA) is the signal that induces the SOS response by promoting the formation of the RecA filament, which in turn activates the auto-cleavage activity of LexA and allows expression of several genes [8]. The SOS response is a very orchestrated pathway by which the bacterial cell improves its capacity to inhibit cell division, repair DNA, and express error-prone DNA polymerases to replicate noninstructive DNA lesions [14]. This pathway has been widely studied in Escherichia coli where more than 40 genes are involved [15]. The first stage of SOS response is the expression of genes related to nucleotide excision repair mechanisms (uvrA, uvrB, uvrD, polB, $\operatorname{ruv} A, \operatorname{ruvB}$, and $\operatorname{dinl})$. If the damaged is not repaired the genes responsible for recombination repair mechanisms ( $\operatorname{rec} A$ and $\operatorname{rec} N$ ) are expressed. Finally, if the SOS response is not successful, then the sulA and umuDC genes are expressed. SulA inhibits cell division and the $u m u D C$ operon encodes the error-prone DNA polV crucial in translesion error-prone DNA synthesis. When sulA is expressed in the late stage of the SOS gene expression, it arrests cell division by binding FtsZ and provides extra time for the mutagenic error-prone polymerases to acquire mutations that allow cells to escape from the metabolic and genomic stress [8, 16-18].

Some difference may be found in the SOS response for each species. For example, this process in Bacillus subtilis is mediated by a similar number of genes than in E. coli, however, only seven genes are common for these two bacteria [19]. In the case of Staphylococcus aureus, only sixteen genes have been identified under the control of LexA, between them one error-prone polymerase is designated here as umuC (SACOL1400) $[9,20]$. The SOS pathway plays a critical role in several processes related to pathogenesis of $S$. aureus, such as emergence of antibiotic resistant strains [21], dissemination of virulence factors [22], and increase of the frequency of small colony variants (SCVs) [23]. In this sense, this chapter aims to provide a comprehensive review about the modulatory effects of compounds (natural or synthetics) on SOS response of $S$. aureus. 


\section{Overview of SOS response in Staphylococcus aureus}

Since DNA damage may occur as a result of environmental agents and drugs, the role of SOS pathway in different conditions has been studied in S. aureus [21-23]. Most of these studies have focused on the effects of clinical relevant antibiotics, especially those from fluoroquinolone class, such as ciprofloxacin. The induction of SOS response in this pathogen has been associated with mutagenesis, spread of virulence factors, and formation of small colony variants [22-27]. We discuss some of the papers related to these subjects in the following topics.

\subsection{SOS response affects the expression of virulence factors in Staphylococcus aureus}

To prove this concept, the effects of SOS response in the dissemination of pathogenicity island-encoded virulence factors in staphylococci was evaluated [22]. S. aureus pathogenicity island (SaPI) comprises a large family of highly mobile phage-related chromosomal islands, which carry a range of virulence genes, for example, TSST1 (toxic shock syndrome toxin), SEB (staphylococcal enterotoxin B), and other superantigens [28]. SaPI are widely distributed among Gram-positive bacteria and they are considered as prototypes for the understanding of the mobile mechanisms of pathogenicity islands, since horizontal gene transfer has an extremely important role in bacterial evolution [29]. It was demonstrated that SOS induction (by ciprofloxacin) induced SaPI excision and replication with participation of at least three different temperate phages $(80,11$, and 147$)$. SOS pathway also regulates the replication and high-frequency transfer of this element, as well as of SaPI1. Theses finds suggested that SOS activation by antibiotics may lead to the spread of staphylococcal virulence genes, an unintended consequence [22].

The influence of subinhibitory concentrations (Sub-MIC) of others antibiotics in the induction of SOS response and horizontal transfer of virulence factors in S. aureus was also evaluated [24]. The authors used antibiotics with different action mechanisms such as lactams (ampicillin, penicillin, ceftriaxone, and cloxacillin), macrolide- $\beta$ lincosamide-streptogramin B antibiotics (erythromycin), aminoglycosides (kanamycin), chloramphenicol, and tetracycline. From these drugs, only $\beta$-lactams induced replication of SOS- inducible prophages $80 \alpha$ and $11 \phi$, resulting in SaPIbov1 transfer. The effects of ciprofloxacin and trimethoprim (a folic acid inhibitor from sulfonamide class) on phage induction and expression of phage-encoded virulence factors were evaluated using $S$. aureus strains isolates from patients with cystic fibrosis [25]. This study analyzed the integration of phages into the chromosomal gene coding for $\beta$-hemolysin $(h l b)$, these phages encode for accessory virulence determinants such as staphylokinase (sak; a plasminogen activator essential for bacteria dissemination from clots and abscesses and resistance against human defensins) and enterotoxins [30, 31]. Sub-MIC of both drugs resulted in delysogenization of strains and replication of $h l b$-converting phages in a dose-dependent manner. The involvement of SOS response in phage mobilization was demonstrated by increase of rec $A$ expression. In addition, induction of 13 was directly associated with phage-encoded virulence gene sak [25].

In another study, the expression of type 5 capsular polysaccharide (CP5) in S. aureus was shown to be linked to SOS response [32]. CP5 is one virulence factor that is important for protection 
against phagocytes [33] and it is an attractive candidate for the development of immunotherapies [34]. The production of CP5 is influenced by various environmental agents (carbon dioxide, iron concentration, and specific nutrients) and controlled by a complex regulatory genetic network [35]. Using a transposon-mediated mutagenesis assay, seven genes were identified affecting the production of CP5, including $s b c D$ and $s b c C$ genes [35]. These genes are adjacent forming the $s b c D C$ locus that negatively affects capsule production. Sub-MIC of SOS inducers (ciprofloxacin or mitomycin $\mathrm{C}$ ) promoted the transcription of $s b c D C$ locus and consequently repressed the CP5 production [32]. The authors suggested that this effect of SOS response in capsule expression could be related to (i) energy saving (the energy needed to capsule biosynthesis would be used for DNA reparation); (ii) improvement of adhesion capability (capsule absence would unmask the adhesion proteins present in cell membrane, promoting bacterial infection and thereby avoiding DNA-damaging agents). These results corroborate with previous study which showed that ciprofloxacin increased the expression of fibronectin-binding proteins (FnBPs) in fluoroquinolone-resistant S. aureus strains [36].

\subsection{SOS response and mutagenesis in Staphylococcus aureus}

Apart from its capacity to express virulence factors, S. aureus is extremely able to acquire resistance to virtually any antibiotic. For example, methicillin-resistant S. aureus (MRSA) strains are important etiologic agents of both nosocomial and community infections. It has been shown that Sub-MIC of fluoroquinolone drugs enhances methicillin resistance in community or nosocomial MRSA isolates [26, 37]. Community-associated MRSA isolates (CA-MRSA) grown in tryptic soy broth containing sub-MIC of fluoroquinolone (ciprofloxacin or levofloxacin) showed increased resistance in nafcillin agar, and this effect was dosedependent. Through microarray analysis it was possible to conclude that alterations-induced fluoroquinolone drugs were mediated by SOS response [26]. In the same context, a later study evaluated the effects of Sub-MIC of ciprofloxacin in the development of rifampin resistance in methicillin-susceptible S. aureus (MSSA) and MRSA strains. Ciprofloxacin induced higher frequencies of rifampin-resistant mutants. A significant proportion of these mutants exhibited in-frame deletions or insertions in the rpoB gene at several positions, while those mutants from ciprofloxacin-free cultures essentially showed single-amino-acid substitutions [27].

\subsection{Induction of SOS increases the frequency of small colony variants in Staphylococcus aureus}

Recently, the activation of SOS response was linked with the enhanced incidence of small colony variants (SCVs) in S. aureus $[11,23]$. S. aureus SCVs are marked by small colony with slow growth phenotype, which is associated with intracellular persistence and reduced antimicrobial susceptibility [38]. S. aureus switch to SCVs phenotype under the pressure of stress elicitors such as oxidative stress [11], cold stress [39], and drug treatment [23]. SCVs are frequently associated with latent or chronic infections, including device-associated infections, bone and tissue infections, and airway infections of cystic fibrosis patients [40].

S. aureus SCVs present mutations in one or few genes related to metabolic pathways resulting in atypical biochemical characteristics [41]. The nature of these mutations is directly 
related to the antibiotic resistance profile exhibited by the SCVs. Resistance to trimethoprimsulfamethoxazole has been associated to alteration on thy $A$ gene, which encodes thymidylate synthase $[42,43]$. This enzyme is essential for DNA biosynthesis as it converts deoxyuridine monophosphate (dUMP) to deoxythymidine monophosphate (dTMP). Diminished concentrations of intracellular dTMP lead to thymidine-dependent SCVs phenotypes (TD-SCVs), which is associated to trimethoprim-sulfamethoxazole resistance and hypermutability [44, 45].

On the other hand, mutations in genes related to menadione or hemin synthesis result in electron transport-defective strains as bacteria are unable to produce menaquinone and cytochromes, respectively [46, 47]. SCVs auxotrophic for menadione or hemin are resistance to aminoglycosides (such as gentamicin) due to a decrease in drug uptake [48]. Fluoroquinolones and mitomycin C Sub-MIC increased the generation of gentamicin-resistant SCVs with an increased mutation rate through activation of the SOS response [23]. The SOS response is also essential for the adaptation of $S$. aureus to oxidative stress, in this case by producing hydrogen peroxide-resistant SCVs [11].

\section{New compounds able to modulate the SOS response in Staphylococcus aureus}

Given the role of SOS response in S. aureus survival and pathogenesis, the effects of new antimicrobial candidates on SOS genes have become more frequently evaluated, especially those which target DNA structure or DNA replication machinery [49, 50]. Gottschalk et al. [49] reported an easy and inexpensive agar-based assay to detect the expression of $r e c A$ induced by a compound. In this assay, a S. aureus $8325-4$ derivative strain carrying the rec $A$ gene fused with the reporter gene lac $Z$ (which encodes for $\beta$-galactosidase) is incorporated in agar plates containing $X$-Gal (5-bromo-4- $\beta$ chloro-3-indolyl-D-galactopyranoside). X-gal is a chromogenic substrate for $\beta$-galactosidase that produces a rich blue color that can easily be detected visually. The compound to be tested should be added in wells in these agar plates and the expression of $\operatorname{rec} A$ is monitored as a blue ring at the point of bacterial growth. Using this assay, the ability of some compound to active SOS response was revealed, such as the lysinepeptoid hybrid LP5 [49], some cyclic peptide inhibitors of the $\beta$-sliding clamp [50], and the amphibian peptide fallaxin analogue FL9 [51]. The induction of SOS response was also related to the anti-S. aureus action of new synthetic bis-indole antibiotics [52]. All these compounds inhibit the DNA replication of $S$. aureus.

Special attention has been given to the use of SOS inhibitors as therapeutic adjuvants in combating bacterial infections. These approaches involve inhibiting the SOS-mediated mutagenesis induced by drugs and thus improving their long-term viability. In these cases, LexA and RecA represent potential targets [53,54]. In fact, the number of SOS inhibitors is still limited and most of the studies use E. coli as model $[55,56]$. Regarding the suppression of SOS response in S. aureus, a study showed baicalein as a potential compound. Baicalein is the main component of the Chinese herb Scutellaria baicalensis Georgi (Labiatae), which shows anti-S. aureus and antioxidant activities [57, 58]. Baicalein inhibited the expression of some SOS genes (recA, lexA, and LexA-regulated DNA polymerase SACOL1400) and the 
rifampin-resistant mutation ratio induced by Sub-MIC of ciprofloxacin. The authors correlated these effects in SOS response with a decrease in the formation of intracellular reactive oxygen species and ATP level after baicalein treatment [59].

In a later study, the effects of novobiocin in the SOS response induced by ciprofloxacin were evaluated. Novobiocin is an aminocoumarin, a class of antibiotics that interferes with ATPase activity of the gyrase subunit B and the topoisomerase IV subunit ParE without inducing double-strand breaks [60-62]. Differently from quinolones, aminocoumarin treatment does not activate SOS response. In fact, novobiocin inhibited the $\operatorname{rec} A$ expression in a LexAindependent manner. Novobiocin was also able to suppress the SOS response induced by ciprofloxacin: it inhibited recA expression and partially reduced the induction of the error-prone polymerase $u m u C$ (regulated by LexA). These effects resulted in a reduction in the frequency of recombination, mutation, and the formation of nonhemolytic variants [20].

The concept that SOS response is a potential target was additionally explored using antimicrobial photoinactivation. Antimicrobial photodynamic therapy (aPDT) is a promising strategy for the treatment of localized infections, such as acne inflammation [63], periodontal, and periimplant diseases [64]. aPDT consists in the use of three elements (photosensitizer agent, visible light, and oxygen), where the damage of different bacterial structures (cell envelopes, lipids, proteins, and DNA) would avoid the development of resistance [65]. The role of DNA damage and SOS response during photoinactivation was recently established. Different exogenous photosensitizers induced DNA damage and consequently the expression of recA. The repression of recA by novobiocin or gene deletion resulted in additional susceptibility of S. aureus toward photoinactivation through increase of DNA damage. These results suggested that the combination of recA inhibitors and photoinactivation could have a clinical relevance [66].

SOS response in E. coli has been shown to be regulated by ribonuclease E (RNase E), an enzyme involved in RNA metabolism (global mRNA degradation, maturation of rRNA, and small regulatory RNA) [67]. RNase E deficient strains exhibit a reduction in SOS activation, revealing that RNase E inhibitors could be possibly used as drug adjuvants [68]. Although RNase E orthologs have been identified in a range of other bacteria and in bacteria and chloroplasts [69, 70], RNA turnover is not regulated by an RNase E ortholog protein in S. aureus [70]. Instead, $S$. aureus has an mRNA degradosome complex formed by diverse proteins, including RNase enzymes (RNase J1, RNase J2, RNase Y, and RnpA), enolase, phosphofructokinase, polynucleotide phosphorylase (PNPase), and DEAD box RNA helicase. RnpA, a component of this complex, has been reported as a target to inhibit bacterial survival and pathogenesis [70, 71]. However, the role of mRNA degradosome complex in SOS response regulation remains to be elucidated in this pathogen.

\section{Conclusion}

The SOS response is an essential pathway for S. aureus survival and pathogenesis. This mechanism is activated by different stress situations (such as environmental alteration, drug, and toxins treatment), which lead to mutagenesis, phenotypical alterations, and spread of 
virulence factors. All these consequences of SOS activation are important to pathogen dissemination and treatment failure. SOS proteins are potential target for therapies, especially those using quinolones and RecA/LexA inhibitors. These studies have shown that SOS inhibitors are able to decrease drug-induced mutagenesis in $S$. aureus. We hope that more researches will be performed in the future to identify more compounds that are able to modulate SOS response, as well as deeper in vivo studies to establish the clinical relevance of them.

\section{Author details}

Luís Cláudio Nascimento da Silva ${ }^{1 *}$, Roseane Costa Diniz ${ }^{1}$, Isana Maria de Souza Feitosa Lima $^{1}$, Camilla Itapary dos Santos ${ }^{1}$, Matheus Silva Alves ${ }^{1}$, Larissa Isabela Oliveira de Souza ${ }^{2}$ and Andrea de Souza Monteiro ${ }^{1}$

*Address all correspondence to: luisclaudionsilva@yahoo.com.br

1 Ceuma University, São Luís, MA, Brazil

2 Research Center Aggeu Magalhães, Recife, PE, Brazil

\section{References}

[1] Jessica MAB, Mark AW, Alison JB, David OO, Laura JVP. Molecular mechanisms of antibiotic resistance. Nature Reviews Microbiology. 2015;13(1):42-51. DOI: 10.1038/ nrmicro3380

[2] Damien R, et al. Fitness cost of antibiotic susceptibility during bacterial infection. Science Translational Medicine. 2015;7(297):297ra114. DOI: 10.1126/scitranslmed.aab1621

[3] Asher B, Ofer F, Orit G, Nathalie QB. Distinguishing between resistance, tolerance and persistence to antibiotic treatment. Nature Reviews Microbiology. 2016;14(5):320-330. DOI: $10.1038 /$ nrmicro.2016.34

[4] World Health Organization. Antimicrobial resistance: global report on surveillance. World Health Organization; Geneva, Switzerland, 2014. 257 p. DOI: http://www.who. int/drugresistance/documents/surveillancereport/en/

[5] Oliver W, et al. Colonisation with multidrug-resistant bacteria is associated with increased mortality in patients with cirrhosis. Gut. 2015;64(7):1183-1184. DOI: 10.1136/ gutjnl-2014-309104

[6] Antoine G, et al. Costs and benefits of high mutation rates: adaptive evolution of bacteria in the mouse gut. Science. 2001;291(5513):2606-2608. DOI: 10.1126/science.1056421

[7] Clara Torres-Barceló, et al. The SOS response increases bacterial fitness, but not evolvability, under a sublethal dose of antibiotic. Proceedings of the Royal Society Biological Sciences. 2015;282:1816. DOI: 10.1098/rspb.2015.0885 
[8] Zeynep B, Didier M. SOS, the formidable strategy of bacteria against aggressions. FEMS Microbiology Reviews. 2014;38(6):1126-1145. DOI: http://dx.doi. org/10.1111/1574-6976.12077

[9] Ryan TC, et al. Complete and SOS-mediated response of Staphylococcus aureus to the antibiotic ciprofloxacin. Journal of Bacteriology. 2007;189(2):531-539. DOI: 10.1128/ JB.01464-06

[10] Steve PB, et al. Starvation, together with the SOS response, mediates high biofilmspecific tolerance to the fluoroquinoloneofloxacin. PLoS Genetics. 2013;9(1):e1003144. DOI: 10.1371/journal.pgen.1003144

[11] Kimberley LP, et al. Staphylococcus aureus adapts to oxidative stress by producing $\mathrm{H}_{2} \mathrm{O}_{2}$-resistant small-colony variants via the SOS response. Infection and Immunity. 2015;83(5):1830-1844. DOI: 10.1128/IAI.03016-14

[12] Didier H, et al. Evidence for induction of integron-based antibiotic resistance by the SOS response in a clinical setting. PLoS Pathogens. 2012;8(6):e1002778. DOI: 10.1371/journal. ppat. 1002778

[13] Hongan L, et al. Antibiotic treatment enhances the genome-wide mutation rate of target cells. Proceedings of the National Academy of Sciences. 2016;113(18):E2498-E2505. DOI: 10.1073/pnas.1601208113

[14] Kelsi LA, et al. Characterization of the Staphylococcus aureusheat shock, cold shock, stringent, and sos responses and their effects on log-phase mRNA turnover. Journal of Bacteriology. 2006;188(19):6739-6756. DOI: 10.1128/JB.00609-06

[15] Justin C, et al. Comparative gene expression profiles following UV exposure in wildtype and SOS-deficient Escherichia coli. Genetics. 2001;158(1):41-64. DOI: http://www. genetics.org/content/158/1/41.long

[16] Bénédicte M. After 30 years of study, the bacterial SOS response still surprises us. PLoS Biology. 2005;3(7):e255. DOI: 10.1371/journal.pbio.0030255

[17] Katharina S, Phuong P, Michael MC, Myron F. Goodman. Roles of DNA polymerase V and RecA protein in SOS damage-induced mutation. Chemical Reviews. 2006;106(2):406419. DOI: $10.1021 /$ cr0404951

[18] Celina J. Inducible SOS response system of DNA repair and mutagenesis in Escherichia coli. International Journal of Biological Sciences. 2008;4(6):338-344. DOI: 10.7150/ ijbs. 4.338

[19] Alexi IG, et al. Characterization of the global transcriptional responses to different types of DNA damage and disruption of replication in Bacillus subtilis. Journal of Bacteriology. 2006;188(15):5595-5605. DOI: 10.1128/JB.00342-06

[20] Wiebke S, Christiane G, Christiane W. Opposing effects of aminocoumarins and fluoroquinolones on the SOS response and adaptability in Staphylococcus aureus. Journal of Antimicrobial Chemotherapy. 2013;68(3):529-538. DOI: 10.1093/jac/dks456 
[21] Arabela C, Konrad P, Adriana ER. Development of homogeneous expression of resistance in methicillin-resistant Staphylococcus aureus clinical strains is functionally associated with a $\beta$-lactam-mediated SOS response. Journal of Antimicrobial Chemotherapy. 2009;64(1):37-45. DOI: 10.1093/jac/dkp164

[22] Úbeda C, Maiques E, Knecht, E, Lasa Í, Novick RP, Penadés JR. Antibiotic-induced SOS response promotes horizontal dissemination of pathogenicity island-encoded virulence factors in staphylococci. Molecular Microbiology. 2005;56(3):836-844. DOI: 10.1111/j.1365-2958.2005.04584.x

[23] Martin V, Wilhelm P, Hanne I. Activation of the SOS response increases the frequency of small colony variants. BMC Research Notes. 2015;8(749):1. DOI: 10.1186/ s13104-015-1735-2

[24] Elisa $\mathrm{M}$, et al. $\beta$-Lactam antibiotics induce the sos response and horizontal transfer of virulence factors in Staphylococcus aureus. Journal of Bacteriology. 2006;188(7):2726-2729. DOI: 10.1128/JB.188.7.2726-2729.2006

[25] Christiane G, Johanna K, Christiane W. Ciprofloxacin and trimethoprim cause phage induction and virulence modulation in Staphylococcus aureus. Antimicrobial Agents and Chemotherapy. 2006;50(1):171-177. DOI: 10.1128/AAC.50.1.171-177.2006

[26] Pierre T, Li B, Henry FC . Subinhibitory fluoroquinolone exposure selects for reduced beta-lactam susceptibility in methicillin-resistant Staphylococcus aureus and alterations in the SOS-mediated response. Research in Microbiology. 2009;160(3):187-192. DOI: 10.1016/j.resmic.2009.03.003

[27] Jean-Philippe D, et al. Impact of ciprofloxacin exposure on Staphylococcus aureusgenomic alterations linked with emergence of rifampin resistance. Antimicrobial Agents and Chemotherapy. 2011;55(5):1946-1952. DOI: 10.1128/AAC.01407-10

[28] Richard PN, Gail EC, Jose RP. The phage-related chromosomal islands of Gram-positive bacteria. Nature Reviews Microbiology. 2010;8:541-551. DOI: 10.1038/nrmicro2393

[29] Carles U, María ÁT-M, José RP, Richard PN. Structure-function analysis of the SaPIbov1 replication origin in Staphylococcus aureus. Plasmid. 2012;67(2):183-190. DOI: 10.1016/j. plasmid.2012.01.006

[30] Douglas RD, Bryan U, Vincent AFi. Uncovering novel mobile genetic elements and their dynamics through an extra-chromosomal sequencing approach. Mobile Genetics Elements. 2016;6(4):e1189987 . DOI: 10.1080/2159256X.2016.1189987

[31] Lena T, Olaf S, Dominique M. Pathogenesis of Staphylococcus aureus blood stream infections. Annual Review of Pathology: Mechanisms of Disease. 2016;11:343-364 . DOI: 10.1146/annurev-pathol-012615-044351

[32] Zhongyi C, Thanh TL, Chia YL. The sbcDC locus mediates repression of type 5 capsule production as part of the SOS response in Staphylococcus aureus. Journal of bacteriology. 2007;189:343-7350. DOI: 1 0.1128/JB.01079-07 
[33] Chia Y L, Jean CL. Staphylococcal Capsule. In: Vicent AF, et al., editor. Gram-Positive Pathogens. 2nd ed. Washington, DC: American Society for Microbiology; 2006. pp. 456-457. DOI: 10.1128/9781555816513.ch37

[34] Ivan AG, et al. Synthesis of Staphylococcus aureus type 5 trisaccharide repeating unit: solving the problem of lactamization. Organic Letters. 2015;17(4):928-931. DOI: 10.1021/ acs.orglett.5b00031

[35] Thanh TL, Chia YL. The arl locus positively regulates Staphylococcus aureus type 5 capsule via an mgrA-dependent pathway. Microbiology. 2006;152(10):3123-3131. DOI: 10.1099/mic.0.29177-0

[36] Carmelo B, et al. Induction of fibronectin-binding proteins and increased adhesion of quinolone-resistant Staphylococcus aureus by subinhibitory levels of ciprofloxacin. Antimicrobial Agents and Chemotherapy. 2000;44(6):1428-1437. DOI: 10.1128/ AAC.44.6.1428-1437.2000

[37] Richard AV, et al. Selection of high-level oxacillin resistance in heteroresistant Staphylococcus aureus by fluoroquinolone exposure. Journal of Antimicrobial Chemotherapy. 2001;48(3):375-381. DOI: 10.1093/jac/48.3.375

[38] Richard AP, et al. Small colony variants: a pathogenic form of bacteria that facilitates persistent and recurrent infections. Nature Reviews Microbiology. 2006;4:295-305. DOI: 10.1038/nrmicro1384

[39] Mousa MA, et al. Metabolomic and proteomic responses of Staphylococcus aureus to prolonged cold stress. Journal of Proteomics. 2015;121:44-55. DOI: 10.1016/j. jprot.2015.03.010

[40] Kahl BC, Becker K, Löffler B. Clinical significance and pathogenesis of staphylococcal small colony variants in persistent infections. Clinical Microbiology Reviews. 2016;29(2):401-427. DOI: 10.1128/CMR.00069-15

[41] Kahl BC. Small colony variants (SCVs) of Staphylococcus aureus-a bacterial survival strategy. Infection, Genetics and Evolution. 2014;21:515-522. DOI: 10.1016/j. meegid.2013.05.016

[42] Besier S, Ludwig A, Ohlsen K, Brade V, Wichelhaus TA. Molecular analysis of the thymidine-auxotrophic small colony variant phenotype of Staphylococcus aureus. International Journal of Medical Microbiology. 2007;297:217-225. DOI: 10.1016/j.ijmm.2007.02.003

[43] Chatterjee I, Kriegeskorte A, Fischer A, Deiwick S, Theimann N, Proctor RA, Peters G, Herrmann M, Kahl BC. In vivo mutations of thymidylate synthase (thy A) are responsible for thymidine dependency in clinical small-colony variants of Staphylococcus aureus. Journal of Bacteriology. 2008;190:834-842. DOI: 10.1128/JB.00912-07

[44] Zander J, Besier S, Saum SH, Dehghani F, Loitsch S, Brade V, Wichelhaus TA. Influence of dTMP on the phenotypic appearance and intracellular persistence of Staphylococcus aureus. Infection and Immunity. 2008;76:1333-1339. DOI: 10.1128/IAI.01075-07 
[45] Besier S, Zander J, Kahl BC, Kraiczy P, Brade V, Wichelhaus TA. The thymidinedependent small-colony-variant phenotype is associated with hypermutability and antibiotic resistance in clinical Staphylococcus aureus isolates. Antimicrobial Agents and Chemotherapy. 2008;52:2183-2189. DOI: 10.1128/AAC.01395-07

[46] McNamara PJ, Proctor RA. Staphylococcus aureus small colony variants, electron transport and persistent infections. International Journal of Antimicrobial Agents. 2000;14:117-122.

[47] Lannergård J, von Eiff C, Sander G, Cordes T, Seggewiss J, Peters G, Proctor RA, Becker K, Hughes D. Identification of the genetic basis for clinical menadione-auxotrophic small-colony variant isolates of Staphylococcus aureus. Antimicrobial Agents and Chemotherapy. 2008;52:4017-4022. DOI: 10.1128/AAC.00668-08.

[48] Dean MA, Olsen RJ, Long SW, Rosato AE, Musser JM. Identification of point mutations in clinical Staphylococcus aureusstrains that produce small-colony variants auxotrophic for menadione. Infection and Immunity. 2014;82:1600-1605. DOI: 10.1128/ IAI.01487-1.

[49] Gottschalk S, Ifrah D, Lerche S, Gottlieb CT, Cohn MT, Hiasa H, Hansen PR, Gram L, Ingmer $\mathrm{H}$, Thomsen LE. The antimicrobial lysine-peptoid hybrid LP5 inhibits DNA replication and induces the SOS response in Staphylococcus aureus. BMC Microbiology. 2013;13(192):1-8. DOI: 10.1186/1471-2180-13-192

[50] Kjelstrup S, Hansen PM, Thomsen LE, Hansen PR, Løbner-Olesen A. Cyclic peptide inhibitors of the $\beta$-sliding clamp in Staphylococcus aureus. PLoS One. 2013;8(9):e72273. DOI: 10.1371/journal.pone.0072273

[51] Gottschalk S, Gottlieb CT, Vestergaard M, Hansen PR, Gram L, Ingmer H, Thomsen LE. Amphibian antimicrobial peptide fallaxin analogue FL9 affects virulence gene expression and DNA replication in Staphylococcus aureus. Journal of Medical Microbiology. 2015;64:1504-1513. DOI: 10.1099/jmm.0.000177

[52] Opperman TJ, Kwasny SM, Li JB, Lewis MA, Aiello D, Williams JD, Peet NP, Moir DT, Bowlin TL, Long EC. DNA targeting as a likely mechanism underlying the antibacterial activity of synthetic Bis-indole Antibiotics. Antimicrobial Agents and Chemotherapy. 2016; DOI: 10.1128/AAC.00309-16

[53] Culyba MJ, Mo CY, Kohli RM. Targets for combating the evolution of acquired antibiotic resistance. Biochemistry. 2015;54(23):3573-3582. DOI: 10.1021/acs.biochem.5b00109

[54] Mo CY, Manning SA, Roggiani M, Culyba MJ, Samuels AN, Sniegowski PD, Goulian M, Kohli RM. Systematically altering bacterial SOS activity under stress reveals therapeutic strategies for potentiating antibiotics. mSphere. 2016;1(4). DOI: 10.1128/ mSphere.00163-16

[55] Sexton JZ, Wigle TJ, He Q, Hughes MA, Smith GR, Singleton SF, Williams AL, Yeh LA. Novel inhibitors of E. coli RecA ATPase activity. Current Chemical Genomics and Translational Medicine. 2010;4:34-42. DOI: 10.2174/1875397301004010034 
[56] Peterson EJ, Janzen WP, Kireev D. Singleton SF high-throughput screening for RecA inhibitors using a transcreener adenosine 5'-O-diphosphate assay. ASSAY and Drug Development Technologies. 2012;10(3):260-268. DOI: 10.1089/adt.2011.0409

[57] Fujita M, Shiota S, Kuroda T, Hatano T, Yoshida T, Mizushima T, Tsuchiya T. Remarkable synergies between baicalein and tetracycline, and baicalein and $\beta$-lactams against methicillin-resistant Staphylococcus aureus. Microbiology and Immunology. 2005;49(4):391-396. DOI: 10.1111/j.1348-0421.2005.tb03732.x

[58] Sahu BD, Kumar JM, Kuncha M, Borkar RM, Srinivas R, Sistla R. Baicalein alleviates doxorubicin-induced cardiotoxicity via suppression of myocardial oxidative stress and apoptosis in mice. Life Sciences. 2016;144(1):8-18. DOI: 10.1016/j.lfs.2015.11.018

[59] Peng Q, Zhou S, Yao F, Hou B, Huang Y, Hua D, Zheng Y, Qian Y. Baicalein suppresses the SOS response system of Staphylococcus aureus induced by ciprofloxacin. Cellular Physiology and Biochemistry. 2011;28(5):1045-1050 . DOI: 10.1159/000335791

[60] Alt S, Mitchenall LA, Maxwell A, Heide L. Inhibition of DNA gyrase and DNA topoisomerase IV of Staphylococcus aureus and Escherichia coli by aminocoumarin antibiotics. Journal of Antimicrobial Chemotherapy. 2011;66(9):2061-2069. DOI: 10.1093/jac/dkr247

[61] Schröder W, Bernhardt J, Marincola G, Klein-Hitpass L, Herbig A, Krupp G, Nieselt K, Wolz C. Altering gene expression by aminocoumarins: the role of DNA supercoiling in Staphylococcus aureus. BMC Genomics. 2014;15;291. DOI:10.1186/1471-2164-15-291

[62] Heide L. New aminocoumarin antibiotics as gyrase inhibitors. International Journal of Medical Microbiology. 2014;304(1):31-36. DOI: 10.1016/j.ijmm.2013.08.013

[63] Jeon YM, Lee HS, Jeong D, Oh HK, Ra KH, Lee MY. Antimicrobial photodynamic therapy using chlorin e6 with halogen light for acne bacteria-induced inflammation. Life Sciences. 2015;124:56-63. DOI: 10.1016/j.lfs.2014.12.029

[64] Al Habashneh R, Asa'ad FA, Khader Y. Photodynamic therapy in periodontal and periimplant diseases. Quintessence International. 2015;46:677-690. DOI: 10.3290/j.qi.a34078.

[65] Almeida A, Faustino MA, Tomé JP. Photodynamic inactivation of bacteria: finding the effective targets. Future Medicinal Chemistry. 2015;7(10):1221-1224. DOI: 10.4155/ fmc.15.59

[66] Grinholc M, Rodziewicz A, Forys K, Rapacka-Zdonczyk A, Kawiak A, Domachowska A, Golunski G, Wolz C, Mesak L, Becker K, Bielawski KP. Fine-tuning recA expression in Staphylococcus aureus for antimicrobial photoinactivation: importance of photoinduced DNA damage in the photoinactivation mechanism. Applied Microbiology and Biotechnology. 2015;99(21):9161-9176. DOI: 10.1007/s00253-015-6863-z

[67] Mildenhall KB, Wiese N, Chung D, Maples VF, Mohanty BK, Kushner SR. RNase E-based degradosome modulates polyadenylation of mRNAs after Rho-independent transcription terminators in Escherichia coli. Molecular Microbiology. 2016;101(4):645-655. DOI: 10.1111/mmi.13413. 
[68] Manasherob R, Miller C, Kim KS, Cohen SN. Ribonuclease E modulation of the bacterial SOS response. Plos One. 2012;7(6):e38426. DOI: 10.1371/journal.pone.0038426.

[69] Aït-Bara S, Carpousis AJ. RNA degradosomes in bacteria and chloroplasts: classification, distribution and evolution of RNase E homologs. Molecular Microbiology. 2015;97(6):1021-1135. DOI: 10.1111/mmi.13095

[70] Eidem TM, Lounsbury N, Emery JF, Bulger J, Smith A, Abou-Gharbia M, Childers W, Dunman PM. Small-molecule inhibitors of Staphylococcus aureusRnpA-mediated RNA turnover and tRNA processing. Antimicrobial Agents and Chemotherapy. 2015;59(4):2016-2028. DOI: 10.1128/AAC.04352-14

[71] Olson PD, Kuechenmeister LJ, Anderson KL, Daily S, Beenken KE, Roux CM, Reniere ML, Lewis TL, Weiss WJ, Pulse M, Nguyen P, Simecka JW, Morrison JM, Sayood K, Asojo OA, Smeltzer MS, Skaar EP, Dunman PM. Small molecule inhibitors of Staphylococcus aureus RnpA alter cellular mRNA turnover, exhibit antimicrobial activity, and attenuate pathogenesis. PLoS Pathogens. 2011;7(2): e1001287. DOI: 10.1371/journal.ppat.1001287. 
